\title{
Workshop on Formal Methods in Human Computer Interaction
}

\author{
Benjamin Weyers \\ RWTH Aachen \\ Germany \\ weyers@vr.rwth- \\ aachen.de
}

\author{
Judy Bowen \\ Waikato University \\ New Zealand \\ jbowen \\ @cs.waikato.ac.nz
}

\author{
Alan Dix \\ University of \\ Birmingham \\ United Kingdom \\ alan@hcibook.com
}

\author{
Philippe Palanque \\ IRIT-ICS, Université \\ Paul Sabatier - \\ Toulouse III \\ palanque@irit.fr
}

\begin{abstract}
This workshop aims to gather active researchers and practitioners in the field of formal methods for interactive systems. The main objective is twofold: on one hand look at the evolutions of the definition and use of formal methods for interactive systems since the last book on the field nearly 20 years ago [1] following the seminal work reported in [2]. On the other hand, to identify important themes for the next decade of research. Formal methods aid in the design, development and evaluation of interactive systems providing the unique opportunity for complete and unambiguous description amenable to formal verification. The HCI community has demonstrated that the next generation of user interfaces is moving off the desktop: these emerging interfaces exploit novel input techniques such as tangible, haptic, camera-based, brain-computer, interaction, present a large quantity of information possibly distributed to a wide range of devices. In this workshop, we will discuss common themes, conflicting approaches and techniques, and future directions for the next generation of formal methods that will support the development of large scale dependable and usable interactive systems.
\end{abstract}

\section{Author Keywords}

formal methods; formal description techniques; formal analysis; verification; usability.

\section{ACM Classification Keywords}

H.5.m. Information interfaces and presentation (e.g., HCI): Miscellaneous.

\section{INTRODUCTION AND DESCRIPTION OF WORK}

The main goal of this workshop is to offer an exchange platform for scientists who are interested in the formal

Permission to make digital or hard copies of part or all of this work for personal or classroom use is granted without fee provided that copies are not made or distributed for profit or commercial advantage and that copies bear this notice and the full citation on the first page. Copyrights for thirdparty components of this work must be honored. For all other uses, contact the Owner/Author.

Copyright is held by the owner/author(s).

EICS'15, June 23-26, 2015, Duisburg, Germany

ACM 978-1-4503-3646-8/15/06.

http://dx.doi.org/10.1145/2774225.2777460 modeling and description of interaction, user interfaces, and interactive systems. It further picks up the notion of a CHI workshop in 1996 [1] addressing an update on the work that has been done since then. Formal modeling in the description of interaction, user interfaces, and interactive systems is still of main interest in current research and modeling strategies. Formal models offer advantages like computer-based validation and verification, formal modification capabilities, and could have capabilities to be executable. Nevertheless, formal modeling underwent various discussions identifying disadvantages, such as inflexibility, high modeling effort, high learning curve, or inefficiency in a wider sense. The workshop is intended to discuss existing formal modeling methods in this area of conflict. Therefore, the participants will be asked to present their perspectives, concepts, and techniques for formal modeling by means of one of two case studies or independently from these in a way the authors choose. The solutions should present but is not restricted to the following aspects: formal modeling approaches, formal verification techniques, formalization of adaptivity and adaption as well as the generation of user interfaces, etc.

The recommended (but not mandatory) use cases define a basic structure for the workshop and further deal as seed for discussions during the workshop. Every participant will have similar knowledge of the use cases and thereby enable them to identify differences between the presented approaches, which finally leads to a complete image of the participating concepts. The use cases will further focus on two different research questions relevant in the intended context. Therefore, the first use case presents a scenario, which focus on controlling automated technical systems. Automation is still a relevant issue in research on human computer interaction [3]. The second use case focus on multi-user interaction, which addresses systems that involve not only single persons and users but whole teams [4].

\section{Use Case 1 - Control of a nuclear power plant}

This first use case focusses on the control of a nuclear power plant, which is partially automated. The main task of the reactor operator is to drive the reactor in a safe state to produce electrical energy. The automated observation system kicks in when the power plant leaves the safe state 
and stepwise suspends the user from being able to control the system and takes over to lead the system back into a safe state. Thus, the more critical the problem is, the more automated the control gets. The main problem to be addressed here is that the human reactor operator has to be in the control loop to enable him to be capable of acting in any control situation correctly as far as he is able to do regarding a complete manual control.

Workshop participants will be asked to approach this use case out of their own perspective. Independent of which perspective is chosen, the focus should be the handling of automation. A detailed description of the process and its automation that are relevant for this use case are described on the workshop's web page ${ }^{1}$, which further includes technical details. This page further provides a simplified simulation implementation of this nuclear plant model. It will be provided to the participants to support them to prepare their solution but will not be obligatory.

\section{Use Case 2 - Air Traffic Management Arrival Manager}

The second case study focuses on the AMAN tool and the EXC_TMA i.e. Executive Controller in the TMA (Terminal Manoeuvring Area). The TMA is the area where are controlled flights approaches and departures in the airspace close to the airport. The AMAN (Arrival MANager) tool is a software planning tool suggesting to the air traffic controller an arrival sequence of aircraft and providing support in establishing the optimal aircraft approach routes. Its main aims are to assist the controller to optimize the runway capacity (sequence) and/or to regulate/manage (meter) the flow of aircraft entering the airspace, such as a TMA [4]. The AMAN tool uses the flight plan data, the radar data, and an aircraft performance model to provide air traffic controllers, via electronic display, two kind of information: 1) A Sequence List (SEQ_LIST), an arrival sequence that optimizes the efficiency of trajectories and runway throughput; 2) Delay management Advisories, for each aircraft in the controller's (ATCO) airspace. One or more EXC_TMA interact with ATCO and communicates with pilots and releases clearances to aircrafts. He/she/they has/have the tactical responsibility of the operations and he/she/they execute the AMAN advisories to sequence aircraft according to the sequence list.

As for case study one, the participants can approach this case study at their will but focusing on the multi-user scenario in this use case. More detailed information such as displayed information and interaction with AMAN is available on the workshop website ${ }^{1}$.

\section{Target Audience}

The target audience are scientist interested in and working on formal modeling and description concepts for interaction, user interfaces, and interactive systems. The goal is to bring various people together not only focusing on formalizing of artifacts for interaction but also concentrating on task and user modeling related to the previous mentioned focuses of the workshop.

\section{WORKSHOP ORGANIZATION}

The workshop is separated into three phases: submission of a max 6 pages paper before the workshop starting a reviewing process, presentation of the accepted work at EICS in the workshop session, and finally all accepted works will be invited to a post-workshop submission of an extended version, which is again reviewed for publication in a Springer book within the HCI Series.

Authors will prepare a description of the formal notation they propose, which could be followed by a description of its application to one of the previously described case studies or a different exemplary description chosen by the authors. These submissions will be reviewed by the program committee members of the workshop and feedback will be sent to the authors. The accepted authors (at least one) will be required to attend the workshop to present their contribution. All presenters will be encouraged to show their solutions in live demos, using modeling tools and formal analysis techniques so that all participants are able to gain insight to the various modeling perspectives and concepts. Nevertheless, a presentation of the submission will be required. All authors attending the workshop will be invited to extend their initial submission based on reviewer's comments and feedback from discussions and questions gathered during the workshop and to submit it to the workshop organizers with the aim of being included after a further reviewing process in the Springer book (approval from Springer as already been obtained). The workshop organizers will edit the book providing an introductory chapter summarizing the objectives, goals, and use cases for the workshop. A conclusive chapter summarizing the various contributions and positioning them according to various criteria which will be identified on the workshop will be co-authored by the authors of chapters who are willing to invest in that activity.

\section{REFERENCES}

1. P. Palanque \& F. Paternó (Ed.). 1997. Formal Methods in Human-Computer Interaction (1st ed.). Springer-Verlag New York, Inc., Secaucus, NJ, USA.

2. M. Harrison and H. Thimbleby (Eds.). 1990. Formal Methods in Human-Computer Interaction. Cambridge University Press, New York, NY, USA.

3. R. Parasuraman, T. Sheridan, C. Wickens: A model for types and levels of human interaction with automation. IEEE Transactions on Systems, Man, and Cybernetics, Part A 30(3): 286-297 (2000)

4. EUROCONTROL. 2010. Arrival Manager. Implementation GUIDELINES and Lessons Learned. Edition 0.1.

${ }^{1} \mathrm{https}: / /$ sites.google.com/site/wsfomchi/ 